\title{
Seroprevalence of Toxoplasma gondii infection and associated risk factors among pregnant women in Debre Tabor, Northwest Ethiopia
}

\author{
Birhan Agmas $^{1}$, Reta Tesfaye ${ }^{2}$ and Digsu Negese Koye ${ }^{3 *}$
}

\begin{abstract}
Background: Toxoplasma gondii is an obligate intracellular protozoan organism that infects both birds and mammals. Human infections are particularly serious if they occur during pregnancy and may result in abortion or congenitally acquired disorders which primarily affect the central nervous system. This study assessed seroprevalence of Toxoplasma gondii infection and associated risk factors among pregnant women at Debre Tabor, Northwest Ethiopia.

Methods: An institution based cross-sectional study was conducted from February to May, 2013. A total of 263 pregnant women who came to Debre Tabor public health facilities for antenatal care were selected and included in the study. The venous blood serum was tested using toxolatex agglutination test. Data on socio-demographic and potential risk factors were collected using structured questionnaire through face-to-face interview. Data were entered and analyzed using SPSS version 20.0. Both bivariate and multivariate analyses were carried out to identify associations between dependent and independent variables.
\end{abstract}

Results: Of 263 pregnant women included in the study, 180 (68.4\%, 95\% Cl: 63.1-71.4\%) were found to be seropositive for anti-toxoplasma antibody. Multivariable analysis showed; age group $\geq 36$ years (Adjusted Odds Ratio $[\mathrm{AOR}]=3.56$;

95\% Cl: 1.01-12.5), cannot read and write ( $\mathrm{AOR}=4.77 ; 95 \% \mathrm{Cl}$ : 1.01-30.3), and cat ownership ( $\mathrm{AOR}=3.36 ; 95 \% \mathrm{Cl}$ : 1.39-8.12) were significantly associated with seropositivity of T.gondii infection.

Conclusions: Seroprevalence of T.gondii infection in Debre Tabor town was high. Age, educational status and presence of cats in home were identified as factors associated with T.gondii infection. Education of pregnant women about the transmission and prevention methods of this infection through health extension and in antenatal care clinics is important. Besides, studies on incidence of toxoplasmosis in newborns and infants are recommended.

Keywords: Seroprevalence, Toxoplasma gondii, Risk factors, Pregnant women, Ethiopia

\section{Background}

Toxoplasma gondii is an obligate intracellular protozoan organism that can cause toxoplasmosis. T.gondii has large number of intermediate hosts including all warm blooded animals and humans. The feline species is the definitive host for this parasite. Cat complete the coccidian life cycle with intestinal replication and passage of oocysts in the feces $[1,2]$.

Humans are commonly infected by ingestion of raw or partly cooked meat containing Toxoplasma viable tissue

\footnotetext{
* Correspondence: digsuneg@gmail.com

${ }^{3}$ Department of Epidemiology and Biostatistics, College of Medicine and

Health Sciences, University of Gondar, Gondar, Ethiopia

Full list of author information is available at the end of the article
}

cysts or by consumption of contaminated food and water with oocysts of $T$. gondi. It can also occur through placental transmission to the fetus $[2,3]$.

After accidental infection of $T$. gondii, immunecompetent adults and children are usually asymptomatic or cause a mild flu-like illness. Recent studies have linked toxoplasmosis with change in behavior and may be a causative or contributory factor in various psychiatric disorders such as depression, anxiety and schizophrenia [4]. However, in immune-compromised people, such as AIDS patients or pregnant women may become seriously ill, and it can occasionally be fatal. It causes severe encephalitis and neurologic diseases, and can affect the heart, liver, inner ears, and eyes (chorioretinitis). In 
congenitally acquired infection; a wide variety of manifestations in the fetus and infant including spontaneous abortion, still-birth, a newborn with hydrocephalus or microcephalus, cerebral calcifications and retinochoroiditis; those leads to mental retardation, blindness, epilepsy, and death. The high prevalence of Toxoplasma infection and its severe consequences in fetus and infant proves the importance of toxoplasmosis in pregnant women [5,6]. Antibiotic treatment to prevent fetal infection and/or fetal complications has not been very effective and the most effective way to prevent congenital Toxoplasmosis is primary prevention [7].

T. gondii infections during pregnancy are mostly diagnosed by detecting anti-T. gondii-specific IgM and IgG antibodies serologically and avidity of $T$. gondii-specific IgG antibodies. Serological tests, such as the latex agglutination (LA) test, Enzyme-linked Immunosorbent Assay (ELISA), and immunofluorescence antibody test (IFAT) are used in many clinical laboratories $[6,8,9]$. Latex agglutination test is relatively rapid and it does not require complex laboratory facilities. It has also high specificity (95\%) and sensitivity (92\%) compared to the gold standard (the Sabin-Feldman dye test). Hence it has been widely used in remote areas in developing countries $[10,11]$.

Seroprevalence of toxoplasmosis varies from country to country. For instance, in Europe, seroprevalences of T. gondii infections in pregnant women vary from $9 \%$ to 67\% [12]. Studies conducted in Asian countries indicated $0.8 \%$ to $28.3 \%$ seroprevalence among pregnant women $[13,14]$. In Africa, studies in Sudan, Burkina Faso and Cameroon showed that $25.3 \%, 34.1 \%$ and $70 \%$ of the pregnant women had anti-T. gondii antibodies respectively [15-17]. In Ethiopia, seroprevalence of $83.6 \%$ from Southwest [18] and $86.4 \%$ from central part of the country [19] were reported among pregnant women.

Studies on the prevalence of toxoplasmosis among pregnant women and associated risk factors are scanty in Ethiopia and unavailable in the study area. Therefore, this study was aimed to estimate the seroprevalence of toxoplasmosis and its associated risk factors among pregnant women attending antenatal care (ANC) at Debre Tabor town, Ethiopia.

\section{Methods}

\section{Study design and area}

An institution based cross-sectional study was conducted from February to May, 2013 in the three health centers and one hospital found in Debre Tabor town. Debre Tabor is 653 kilometers northwest of Addis Ababa, the capital of Ethiopia. This town is situated at latitude of $11.8500^{\circ}$ North; longitude $38.0167^{\circ}$ East and an altitude of 2,706 meters above sea level. It has a total population of 71,100 of which 35,372 are males and
35,728 are females [20]. Its temperature ranges from $15^{\circ}$ $\mathrm{C}$ to $18.5^{\circ} \mathrm{C}$.

\section{Study Population}

The source populations were pregnant women who live in the catchment area of Debre Tabor administration. Study population comprised of all pregnant women who attended their ANC follow up at Debre Tabor public health facilities. The study participants were those pregnant women who attend ANC at these health facilities.

\section{Sample size and sampling technique}

A single population proportion formula was used to estimate the sample size [21]. The following assumptions were made; $95 \%$ confidence level, $5 \%$ margin of error and seroprevalence of $T$. gondii among pregnant women of $83.6 \%$ from previous study in Jimma [18] and 25\% for expected non-response rate. Computing with the above formula gives a total sample size of 263. About 5800 pregnant women are expected to attend ANC annually in the administration. One month data from the ANC registration book of the health institutions was taken to approximate the total number of pregnant women attending ANC during the study period and it was estimated that 966 women will attend ANC in study period. Then systematic random sampling method was used with sampling interval of 3 to select the study participants.

\section{Sample collection, transport and laboratory examination}

Blood samples were collected from study participants who visited the health facilities for antenatal care during the study period. Three $\mathrm{ml}$ of venous blood was taken with plain tube by strictly following standard operational procedures. The specimens were transported to Debre Tabor General Hospital laboratory using an ice bag with ice packs. The serum was separated from the other blood constituents after clotting by centrifugation at $5000 \mathrm{rpm}$ for 10 minutes. The samples were kept at $-20^{\circ} \mathrm{C}$ till serologically tested. The serological test was done by Latex agglutination slide test. This test detects antibodies of $T$. gondii from acute (first 1-2 weeks) to lifelong previous infection. The test reagent is standardized to detect more than $10 \mathrm{IU} / \mathrm{ml}$ anti-Toxoplasma antibodies. The presence or absence of a visible agglutination indicates the presence or absence of anti-Toxoplasma antibodies in the samples tested, but it was not possible to distinguish between IgG and IgM. The test was done according to the manufacturer's instruction (Joaquim Costa, 18, 2a planta. 08390 Montgat-Barcelona (Spain).

\section{Questionnaire survey}

Information on socio-demographic data such as age, educational level, birth place, residence place, occupation and 
history of exposure for the possible associated risk factors like consumption of raw meat and vegetables, contact with cat, cat ownership, contact with soil, HIV status and other valuable information were collected by interview with structured questionnaire.

\section{Data management and analysis}

Data obtained from both the questionnaire and laboratory tests were entered and analyzed using the statistical software SPSS version 20.0. Descriptive statistics was used to summarize the data. Variance inflation factor (VIF) was used to check multicollinearity problem. Variables which have VIF value greater than 10 are considered to be collinear variables. However, in the data set no collinearity problem was observed. Bivariate logistic regression was used primarily to check for variables that have association with the dependent variable. Variables found to have a p-value of $<0.2$ in bivariate analysis were fitted into multivariable logistic regression model for controlling the possible effect of confounders and finally variables which had significant association were identified on the basis of $95 \%$ confidence interval. A p-value of less than 0.05 was taken as cut of point to declare significant association. Odds ratio was used in assessing strength of association.

\section{Operational definition of variables}

Close contact with cat was defined as any practices that get in touch with cats or their feces such as playing with cat, clean/touch cat litter box, clean their litter and give feed for cats.

\section{Ethical considerations}

The study protocol was reviewed and approved by the Institution Review Board of Institute of Public Health, College of Medicine and Health Sciences, University of Gondar (Ref.No SPH/1568/06/05). Official permission was also obtained from the respective bodies at Amhara Regional Health Bureau and Debre Tabor health facilities. Verbal informed consent was obtained from each study participant after being informed about the study. Participation was purely voluntary and the participants have the right to know their results. The information was kept confidentially and anonymous test was done. Treatment was given for pregnant women who were coinfected with HIV following standard protocols of the Federal Ministry of Health (FMOH) of Ethiopia [22].

\section{Results}

Socio-demographic characteristics of pregnant women

A total of 263 pregnant women were included in the analysis. The age range of the pregnant women studied was from 18-44 years (mean age of 29.56 years and $\mathrm{SD} \pm 6.42$ ). Seventy five $(28.5 \%)$ of them attended secondary education.
Majority of the pregnant women, 212 (80.6\%) were urban residents and 145 (55.1\%) were born at rural areas. Of the total study participants, 189 (71.9\%) were house wives in occupation (Table 1).

\section{Behavioral characteristics of pregnant women}

Two hundred fifty one antenatal care followers had a history of exposure for one or more well-known predictor/risk factors for Toxoplasma infection. Sixty two $(23.6 \%)$ of the pregnant women eat raw meat and 118 (44.9\%) of them had cats in their home. Nearly one quarters of the pregnant women (27\%) had contact with cats and garden soil (Table 2).

Clinical and obstetrical characteristics of pregnant women During the time of this study, only one pregnant woman had history of blood transfusion. Two participants were found to be HIV positive; they know their HIV status during the routine HIV testing service at the ANC clinic. None of the women had history of organ transplantation. Out of 263 study participants, 102 (38.8\%) were in third trimester of pregnancy. Eighty five (32.3\%) of the pregnant women were nulligravidae and the remaining were multigravidae.

\section{Seroprevalence of $T$. gondii among pregnant women} Out of 263 samples tested, the seroprevalence of T.gondii was $180(68.4 \%)$ within 95\% confidence interval of $63.1 \%-71.4 \%$.

Table 1 Socio-demographic characteristics of pregnant women in Debre Tabor, Northwest; Ethiopia

\begin{tabular}{llll}
\hline Variables & & Frequency & Percent (\%) \\
\hline Age & $16-20$ & 32 & 12.2 \\
& $21-25$ & 50 & 19.0 \\
& $26-30$ & 67 & 25.5 \\
& $31-35$ & 63 & 24.0 \\
Educational level & Cannot read and write & 26 & 19.4 \\
& Can only read and write & 40 & 9.9 \\
& Primary & 53 & 20.2 \\
& Secondary & 75 & 28.5 \\
Birthplace & Tertiary & 69 & 26.2 \\
& Urban & 145 & 55.1 \\
Residence place & Rural & 118 & 44.9 \\
& Urban & 212 & 80.6 \\
occupation & Rural & 51 & 19.4 \\
& Government employee & 65 & 24.7 \\
& House wife & 189 & 71.9 \\
& others* & 9 & 3.4 \\
\hline
\end{tabular}

*Merchants, private employee, students. 
Table 2 Behavioral characteristics of pregnant women in DebreTabor, Northwest Ethiopia

\begin{tabular}{|c|c|c|}
\hline Variables & Frequency & Percent (\%) \\
\hline \multicolumn{3}{|l|}{ Eat raw meat } \\
\hline Yes & 62 & 23.6 \\
\hline No & 201 & 76.4 \\
\hline \multicolumn{3}{|c|}{ Eat dried/cured meat } \\
\hline Yes & 24 & 9.1 \\
\hline No & 239 & 90.9 \\
\hline \multicolumn{3}{|c|}{ Eat raw vegetable } \\
\hline Yes & 44 & 16.7 \\
\hline No & 219 & 83.3 \\
\hline \multicolumn{3}{|c|}{ Ownership of cat } \\
\hline Yes & 118 & 44.9 \\
\hline No & 145 & 55.1 \\
\hline \multicolumn{3}{|c|}{ Close contact with cat } \\
\hline Yes & 71 & 27.0 \\
\hline No & 192 & 73.0 \\
\hline \multicolumn{3}{|c|}{ Source of water } \\
\hline Tap water & 211 & 80.2 \\
\hline Other source* & 52 & 19.8 \\
\hline \multicolumn{3}{|c|}{ Contact with garden soil } \\
\hline Yes & 71 & 27.0 \\
\hline No & 192 & 73.0 \\
\hline
\end{tabular}

*dug-wells, spring water sources.

\section{Factors associated with seropositivity}

In the bivariate analysis, some variables such as age, residence, educational status, occupation, ownership of cat, close contact with cat and number of children were identified as possible risk factors associated with $T$. gondii infection. Multivariable logistic regression analysis showed that age, educational level and cat ownership were significantly associated with seropositivity of T.gondii infection (Table 3). Accordingly, pregnant women in the age range of $31-35$ years and 36 years and above were 5.50 ( 95\% CI: 1.67, 17.97) and 3.56 (95\% CI: 1.01,12.51) times more likely to be seropositive respectively as compared to women in the age range of $16-20$ years. Educational status of pregnant women was identified as one of the risk factors for seropositivity of $T$. gondii. Those women who cannot read and write were 4.77 (95\% CI: $1.01,30.3)$ times more likely to be seropositive as compared to those who attend tertiary education. Those women who had cats at their home were 3.36 (95\% CI: $1.39,8.12$ ) times more likely to be seropositive as compared to those who didn't have cats at their home.

\section{Discussion}

In this institution based cross-sectional study, the data showed a toxo-latex agglutination test seroprevalence of
68.4\% among pregnant women in Debre Tabor town, Ethiopia. This result is much higher than those reported in pregnant women from other countries like Korea 0.8\%, Sudan 25.5\% and Burkina Faso 34.1\% [13,15,16]. It was similar to the study done in Cameroon that showed seroprevalence of $70 \%$ among pregnant women [17]. The causes for these high seroprevalence variations are attributed to environmental distinctiveness (dry climate, rainfall, temperature, soil type, altitude) and mothers' characteristics such as management of cats, educational level, hygienic practice and feeding habit $[6,23]$.

Former serological study in Ethiopia showed $68.8 \%$ to 92.6\% prevalence in central part and $83.6 \%$ in Southwest part of the country $[18,19]$. The lower seroprevalence found in our study compared with those reported elsewhere might be explained by differences in altitude. The study area has an elevation of 2706 meters above sea level; high altitude does not favor oocyst sporulation and survival. Prevalence of $T$. gondii infection has been found lower in high altitudes as compared to low altitudes [24,25]. Even though it is relatively lower than other studies, $68.4 \%$ shows high burden of toxoplasmosis in the study area. This high prevalence may be due to; presence of large number of cat population and living habit of cats and humans closely, inadequate supply of water for hygienic practice. The above noted factors aid the transmission and survival of T.gondii. The other difference could be also due to the differences in methods used and the sensitivity difference. Two of the previous studies used ELISA which has a sensitivity of 98\% [18,19].

Congenital transmission of $T$. gondi infection is a risk in areas where the disease is endemic [26]. In our finding the high prevalence obtained indicates the need for further study on incidence of this infection among infants and routine screening of women in antenatal care clinics. This study didn't identify whether the infection is acute or not.

Age, educational level, and ownership of cat were identified as possible risk factors associated with $T$. gondii infection. The probability of having T.gondii antibody among pregnant women older than 36 years was 3.56 times higher than those pregnant women of 16-20 year age groups. This is in agreement with other reports from south west part of Ethiopia [18]. In dissimilarity to this finding, studies done in Thailand, Cameroon and Addis Ababa, Ethiopia, showed that seroprevalence and age were not found to have statistically significant association $[14,17,27]$.The significant effect of age on T.gondii serostatus in our study subjects may suggest that the higher age group had long duration of exposure.

Women who cannot read and write were 4.77 times more likely to acquire $T$. gondii infection than those pregnant women who had tertiary level of education. This finding was in accordance with a study done in Brazil 
Table 3 Bivariate and multivariate analyses of factors associated with seroprevalnce of T.gondii among pregnant women, Northwest Ethiopia

\begin{tabular}{|c|c|c|c|c|}
\hline \multirow[t]{2}{*}{ Variables } & \multicolumn{2}{|c|}{ Toxoplasmosis seropositivity } & \multirow[t]{2}{*}{ Crude OR $(95 \% \mathrm{Cl})$} & \multirow[t]{2}{*}{ Adjusted OR $(95 \% \mathrm{Cl})$} \\
\hline & Yes & No & & \\
\hline \multicolumn{5}{|l|}{ Age } \\
\hline $16-20$ & 16 & 16 & 1 & 1 \\
\hline $21-25$ & 25 & 25 & $0.99(0.41,2.43)$ & $0.98(0.32,2.97)$ \\
\hline $26-30$ & 41 & 26 & $1.58(0.67,3.69)$ & $1.59(0.55,4.64)$ \\
\hline $31-35$ & 54 & 9 & $6(2.23,16.13)^{*}$ & $5.50(1.67,17.97)^{*}$ \\
\hline$\geq 36$ & 44 & 7 & $6.29(2.18,18)^{*}$ & $3.56(1.01,12.51)^{*}$ \\
\hline \multicolumn{5}{|l|}{ Educational level } \\
\hline Cannot read and write & 24 & 2 & $5.25(1.14,24.27)^{*}$ & $4.77(1.01,30.3)^{*}$ \\
\hline Read and write only & 33 & 7 & $2.06(0.78,5.41)$ & $1.72(0.58,5.14)$ \\
\hline Primary & 45 & 8 & $2.46(0.99,6.11)$ & $2.04(0.72,5.82)$ \\
\hline Secondary & 30 & 45 & $0.29(0.15,0.58)$ & $0.40(0.16,0.99)$ \\
\hline Tertiary & 48 & 21 & 1 & 1 \\
\hline \multicolumn{5}{|l|}{ Residence place } \\
\hline Urban & 137 & 75 & 1 & 1 \\
\hline Rural & 43 & 8 & $2.94(1.32,6.59)^{*}$ & $1.32(0.44,4.02)$ \\
\hline \multicolumn{5}{|l|}{ Occupation } \\
\hline Government employee & 28 & 37 & 1 & 1 \\
\hline House wife & 146 & 43 & $4.49(2.47,8.15)^{*}$ & $1.64(0.70,3.8)$ \\
\hline Others & 6 & 3 & $2.64(0.61,11.50)$ & $0.91(0.13,6.54)$ \\
\hline \multicolumn{5}{|l|}{ Ownership of cat } \\
\hline Yes & 102 & 16 & $5.48(2.95,10.2)^{*}$ & $3.36(1.39,8.12)^{*}$ \\
\hline No & 78 & 67 & 1 & \\
\hline \multicolumn{5}{|l|}{ Close contact with cat } \\
\hline Yes & 65 & 6 & $7.25(3,17.6)^{*}$ & $2.12(0.67,6.67)$ \\
\hline No & 115 & 77 & 1 & 1 \\
\hline \multicolumn{5}{|l|}{ Source of water } \\
\hline Tap water & 140 & 71 & 1 & \\
\hline Other source & 40 & 12 & $0.59(0.29,1.2)$ & - \\
\hline \multicolumn{5}{|l|}{ Contact with Soil } \\
\hline Have garden & 54 & 17 & $1.66(0.89,3.10)$ & - \\
\hline Have not garden & 126 & 66 & 1 & \\
\hline \multicolumn{5}{|l|}{ Number of children } \\
\hline No child & 40 & 45 & 1 & 1 \\
\hline One child & 64 & 20 & $3.60(1.86,6.95)^{*}$ & $2.22(1.0,4.91)$ \\
\hline Two children & 47 & 13 & $4.07(1.93,8.60)^{*}$ & $1.78(0.67,4.74)$ \\
\hline Three children & 22 & 2 & $12.3(2,55)$ & $2.47(0.36,17.2)$ \\
\hline Four or more & 7 & 3 & $2.62(0.6,10)$ & $0.40(0.04,3.72)$ \\
\hline
\end{tabular}

*Significant at P-Value $<0.05$.

which reported pregnant women with low educational status had higher prevalence of T.gondii antibody [28]. Women who cannot read and write have less hygienic practice and they are more likely to be rural residents. They might have garden/agriculture and they may have close contact with animals as compared to the educated ones.

In this cross sectional study, ownership of cat was associated with seropositivity of T.gondii. Pregnant women who had cats at home were 3.36 times more likely to be 
seropositive for $T$. gondii compared with those who did not have. This finding is in agreement with reports from south west and central parts of Ethiopia, Thailand and China $[14,18,19,29]$. In difference; Cameroonian study found no association between sero-prevalence of T.gondii and ownership of cat [17]. In our study area, cats are kept mainly for the purpose of cleaning rats/mice and as pet animal. Cats are the definitive host where sexual multiplication of T.gondii takes place and excrete the oocysts with feces; due to this they are considered the major source of T.gondii infection to humans and animals [2,3].

Other behavioral risk factors such as; eating raw meat or vegetables, drinking untreated water, and contact with garden soil did not show significant association with seroprevalence of T.gondii in our study.

\section{Limitations}

Further confirmatory tests/tests that can identify the type of antibody to identify acute or latent type of T.gondii were not done. Risk factor assessment was done based on study participants' response which may suffer from recall bias. Since this study was institution based study, it may not represent the general population. As a cross-sectional study, it may suffer from chicken-egg dilemma.

\section{Conclusions}

More than two thirds of the pregnant women were positive for T.gondii infection. This high seroprevalence of T. gondii infection shows the need for preventive measures such as awareness creation about toxoplasmosis; mainly education of pregnant women about the transmission and prevention methods at antenatal care clinics. Age, educational status and presence of cats at home were identified as possible associated risk factors of T.gondii infection. Cat owners, particularly pregnant women, should take necessary preventive measures like not eating raw meat, proper disposal of cat feces and keep hygiene to avoid Toxoplasma infection. Studies that show incidence of T. gondii among the neonates have to be done to introduce routine antenatal screening program to control congenital Toxoplasmosis.

\section{Competing interests}

The authors declare that they have no competing interests.

\section{Authors' contributions}

BA designed the study, collected data, performed the statistical analysis and drafted the manuscript. RT and DNK participated in the study design and manuscript writing. All authors contributed to the data analysis, read and approved the final manuscript.

\section{Acknowledgments}

We want to express our great thanks to health professionals working in the public health facilities of Debre Tabor town for their co-operation during data collection. We are also grateful to the study participants.

\section{Author details}

${ }^{1}$ South Gondar Zone Department of Agriculture and Rural Development, Debre Tabor, Ethiopia. ${ }^{2}$ Department of Veterinary Epidemiology and Public Health, Faculty of Veterinary Medicine, University of Gondar, Gondar, Ethiopia. ${ }^{3}$ Department of Epidemiology and Biostatistics, College of Medicine and Health Sciences, University of Gondar, Gondar, Ethiopia.

Received: 8 August 2013 Accepted: 23 March 2015

Published online: 29 March 2015

\section{References}

1. Smith JE, Reduck NR. Toxoplasma gondii strain variation and pathogenicity. Lancaster, PA: Technnomic Publishing; 2000.

2. Dubey JP, Jones JL. Toxoplasma gondii infection in humans and animals in the United States. Int J Parasitol. 2008;38(11):1257-78.

3. Montoya JG, Remington JS. Principles and practice of infectious Diseases, vol. 185; 2006: 73-82

4. Henriquez SA, Brett R, Alexander J, Pratt J, Roberts CW. Neuropsychiatric disease and Toxoplasma gondii infection. Neuroimmunomodulation. 2009;16(2):122-33.

5. Goldenberg RL, Thompson C. The infectious origins of stillbirth. Am J Obstet Gynecol. 2003;189(3):861-73.

6. Gibbs RS. The origins of stillbirth: infectious diseases. Semin Perinatol. 2002;26(1):75-8.

7. Thiébau R, Leproust S, Chêne G, Gilbert R. Effectiveness of prenatal treatment for congenital toxoplasmosis: a meta-analysis of individual patients' data. Lancet. 2007;369(9556):115-22.

8. Jones JL, Lopez A, Wilson M, Schulkin J, Gibbs R. Congenital toxoplasmosis: a review. Obstet Gynecol Surv. 2001;56(5):296-305.

9. Liesenfeld O, Wong SY JSR, editors. Cecil text book of medicine. 21st ed. Philadelphia, PA,: USA: WB. Saunders; 2000

10. Montoya JG, Liesenfeld O. Toxoplasmosis. Lancet. 2004;363(9425):1965-76.

11. Alvarado-Esquivel C, Sethi S, Janitschke K, Hahn H, Liesenfeld O. Comparison of two commercially available avidity tests for toxoplasma-specific lgG antibodies. Arch Med Res. 2002;33(6):520-3.

12. Cook AJ, Gilbert RE, Buffolano W, Zufferey J, Petersen E, Jenum PA, et al. Sources of toxoplasma infection in pregnant women: European multicentre case-control study. European Research Network on Congenital Toxoplasmosis. BMJ. 2000;321(7254):142-7.

13. Song K-J, Shin J-C, Shin H-J, Nam H-W. Seroprevalence of toxoplasmosis in Korean pregnant women. Korean J Parasitol. 2005;43(2):69-71.

14. Nissapatorn V, Suwanrath C, Sawangjaroen N, Ling LY, Chandeying V. Toxoplasmosis-serological evidence and associated risk factors among pregnant women in southern Thailand. Am J Trop Med Hyg. 2011;85(2):243-7.

15. Elnahas A, Gerais AS, Elbashir MI, Eldien ES, Adam I. Toxoplasmosis in pregnant Sudanese women. Saudi Med J. 2003;24(8):868-70.

16. Simpore J, Savadogo A, Ilboudo D, Nadambega MC, Esposito M, Yara J, et al. Toxoplasma gondii, HCV, and HBV seroprevalence and co-infection among HIV-positive and -negative pregnant women in Burkina Faso. J Med Virol. 2006;78(6):730-3.

17. Njunda AL, Assob JCN, Nsagha DS, Kamga HLF, Nde PF, Yugah VC. Seroprevalence of Toxoplasma gondii infection among pregnant women in Cameroon. J Public Health Africa. 2011;2(2):98-101.

18. Zemene E, Yewhalaw D, Abera S, Belay T, Samuel A, Zeynudin A. Seroprevalence of Toxoplasma gondii and associated risk factors among pregnant women in Jimma town, Southwestern Ethiopia. BMC Infect Dis. 2012;12:337.

19. Gebremedhin EZ, Abebe AH, Tessema TS, Tullu KD, Medhin G, Vitale M, et al. Seroepidemiology of Toxoplasma gondii infection in women of child-bearing age in central Ethiopia. BMC Infect Dis. 2013;13:101.

20. CSA: Summary and statistical report of the 2007 population and housing census. In. Addis Ababa, Ethiopia; 2008.

21. James FJ, David L, Joans GE. Epidemiology, Biostatistics, and preventive medicine. 2nd ed. Philadelphia: Saunders/Elsevier; 2001.

22. $\mathrm{FMOH}$. Guidelines for management of opportunistic infections and anti retroviral treatment in adolescents and adults in Ethiopia. Office Federal Ministry of Health. In: Federal HIV/AIDS Prevention and Control. 2008.

23. Dubey JP, Lindsay DS, Speer CA. Structures of Toxoplasma gondii tachyzoites, bradyzoites, and sporozoites and biology and development of tissue cysts. Clin Microbiol Rev. 1998;11(2):267-99. 
24. Alvarado-Esquivel C, Sifuentes-Alvarez A, Narro-Duarte SG, Estrada-Martínez S, Díaz-García JH, Liesenfeld O, et al. Seroepidemiology of Toxoplasma gondii infection in pregnant women in a public hospital in northern Mexico. BMC Infect Dis. 2006;6:103.

25. Dubey JP. Toxoplasma gondii oocyst survival under defined temperatures. J Parasitol. 1998;84(4):862-5.

26. Dubey JP. Toxoplasmosis: Zoonosis Update. JAVMA. 1994;205:1594-5

27. Shimelis T, Tebeje M, Tadesse E, Tegbaru B, Terefe A. Sero-prevalence of latent Toxoplasma gondii infection among HIV-infected and HIV-uninfected people in Addis Ababa, Ethiopia: A comparative cross-sectional study. BMC Res Notes. 2009;2:213.

28. Bittencourt LHFB, Lopes-Mori FMR, Mitsuka-Breganó R, Valentim-Zabott M, Freire RL, Pinto SB, et al. Seroepidemiology of toxoplasmosis in pregnant women since the implementation of the Surveillance Program of Toxoplasmosis Acquired in Pregnancy and Congenital in the western region of Paraná, Brazil. Rev Bras Ginecol Obstet. 2012;34(2):63-8.

29. Zhou P, Chen Z, Li H-L, Zheng H, He S, Lin R-Q, et al. Toxoplasma gondii infection in humans in China. Parasit Vectors. 2011;4:165.

\section{Submit your next manuscript to BioMed Central and take full advantage of:}

- Convenient online submission

- Thorough peer review

- No space constraints or color figure charges

- Immediate publication on acceptance

- Inclusion in PubMed, CAS, Scopus and Google Scholar

- Research which is freely available for redistribution 